\title{
Kinetic theory of steady chemical nucleation in the gas phase
}

\author{
T. Yamamoto ${ }^{1}$, T. Chigai $^{1}$, S. Watanabe ${ }^{1}$, and T. Kozasa ${ }^{2}$ \\ 1 Department of Earth and Planetary Sciences, Nagoya University, Nagoya 464-8602, Japan \\ 2 Division of Earth and Planetary Sciences, Graduate School of Science, Hokkaido University, \\ Sapporo 060-0810, Japan \\ Received 20 March 2001 / Accepted 4 October 2001
}

\begin{abstract}
We develop a kinetic theory of nucleation involving chemical reactions in the gas phase. For the basis of deriving the chemical nucleation rate, chemical kinetic considerations are presented on the steady current density and the effective rate constants of the overall reaction, which is a sum of a sequential elementary reactions. We formulate the steady rate of chemical nucleation in a multi-component vapor, in which nucleation occurs via the chemical reactions yielding a condensate having a stoichiometric composition. An exact expression of the steady nucleation rate is given together with its approximate formulas for practical applications. The present formulation is not concerned with any particular cluster model. The supersaturation ratio for a many-component vapor is defined so as to be a natural extension of that for a one-component vapor. It is shown that the transition probabilities due to growth and decay of the clusters are of the same form as the growth and evaporation rates in a one-component vapor.
\end{abstract}

Key words. astrochemistry - molecular processes - methods: analytical

\section{Introduction}

Nucleation is one of the important elementary processes of dust formation in astrophysical environments (see e.g., Sedlmayr \& Dominik 1995) as well as of many phenomena studied in various fields of science and engineering. The study of the nucleation theory has concentrated mainly on nucleation in one-component systems, in which vapor and condensate are of the same composition such as in condensation of $\mathrm{H}_{2} \mathrm{O}$ vapor into the liquid droplets. However, it is usual in many condensation phenomena that the composition of condensate is different from that of the vapor as are the cases of silicate and carbon dust formation in circumstellar space and many of the condensation experiments in laboratories (e.g., Nuth et al. 2000 and references therein). There are two cases where the vapor and the condensate are of different compositions: one is the case where the condensate is a mixture such as sulfuric acid-water mixtures in polluted air (see Hirschfelder 1974 and references therein). The other is the case where the condensate is formed through chemical reactions yielding a stoichiometric compound. The theory of nucleation of mixtures was formulated by Rice (1950) for binary systems, and was extended by Hirschfelder (1974) to many-component systems. On the other hand, the theory of chemical nucleation has not been well established.

In the astrophysical context, Gail \& Sedlmayr (1988) made an attempt to formulate chemical nucleation, and

Send offprint requests to: T. Yamamoto, e-mail: ty@eps .nagoya-u.ac.jp proposed a method of calculating numerically the steady nucleation rate involving chemical reactions. The method requires the data of rate constants of the reactions involving clusters of various sizes, but most of the data are unavailable for the materials of interest. The analytic expression of the nucleation rate applicable when one growth species dominates like $\mathrm{C}_{2} \mathrm{H}_{2}$ in carbon dust formation in C-rich AGB stars is essentially equivalent to that for a onecomponent vapor. Kozasa \& Hasegawa (1987) proposed a concept of a key species, which is the species of the least collision frequencies among the reactants relevant to the reactions accompanied with chemical nucleation. They assumed that the nucleation rate was controlled by two-body collisions between the clusters and the molecules of a key species. Chigai et al. (1999) derived a chemical nucleation rate employing the key-species concept. However, the validity of the key-species concept has not been examined.

Girshick (1997) obtained an exact expression of the nucleation rate in view of the nucleation in silane plasmas under conditions typical of amorphous silicon thin film deposition during the fabrication of integrated circuits. The reactions that he supposed are:

$\mathrm{SiH}_{3}^{-}+\mathrm{SiH}_{4} \rightarrow \mathrm{Si}_{2} \mathrm{H}_{5}^{-}+\mathrm{H}_{2}$

$\mathrm{Si}_{2} \mathrm{H}_{5}^{-}+\mathrm{SiH}_{4} \rightarrow \mathrm{Si}_{3} \mathrm{H}_{7}^{-}+\mathrm{H}_{2}$,

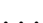

$\mathrm{Si}_{n-1} \mathrm{H}_{2 n-1}^{-}+\mathrm{SiH}_{4} \rightarrow \mathrm{Si}_{n} \mathrm{H}_{2 n+1}^{-}+\mathrm{H}_{2}$, 
which are expressed in general by

$$
\mathrm{A}_{i} \mathrm{~B}_{m+(i-1)(n-p)}+\mathrm{AB}_{n} \rightarrow \mathrm{A}_{i+1} \mathrm{~B}_{m+i(n-p)}+\mathrm{B}_{p}
$$$$
(i \geq 1) \text {. }
$$

The characteristics of this type of reactions are that these are equimolar reactions with only one kind of growth species $\mathrm{AB}_{n}$, and that there is a starting block $\mathrm{AB}_{m}$, which is regarded to be a monomer.

In astrophysical nucleation usually met, however, there is no starting block, and growth species more than one species participate in the reactions like, for example, $\mathrm{TiC}$ formation around C-stars (Chigai et al. 1999) through the reactions such that

$$
\begin{aligned}
& \mathrm{Ti}+\mathrm{Ti}+\mathrm{C}_{2} \mathrm{H}_{2} \rightarrow(\mathrm{TiC})_{2}+\mathrm{H}_{2}, \\
& (\mathrm{TiC})_{i}+\mathrm{Ti}+\frac{1}{2} \mathrm{C}_{2} \mathrm{H}_{2} \rightarrow(\mathrm{TiC})_{i+1}+\frac{1}{2} \mathrm{H}_{2} \quad(i \geq 2) .
\end{aligned}
$$

In this paper, we derive a steady nucleation rate applicable to nucleation involving more general types of chemical reactions than that Girshick (1997) supposed. The nucleation rate that we have obtained is an exact one free from any particular cluster model for the evaluation of the Gibbs free energy of formation of the clusters, and includes no "tuning parameter". Formulation is given for nucleation in ideal gases, but the results will be applicable to nucleation in ideal solutions as well if the rate constants are appropriately taken. Section 2 presents chemical kinetic considerations relevant to the formulation of the nucleation rate. The chemical nucleation rate and its approximate but concise expression are given in Sect. 3 and 5, respectively. The case when monomers are present is discussed in Sect. 4. Section 6 examines the validity of the key-species concept and clarifies a criterion of its applicability. Concluding remarks are presented in Sect. 7.

\section{Chemical kinetic considerations}

We present a chemical kinetic discussion on the current densities (which we call simply currents hereafter) and the rate constants of chemical reactions relevant to the formulation of the steady chemical nucleation rate given in Sect. 3. Chemical kinetics is applicable if elastic collisions dominate over reactive collisions in the Boltzmann equation, in other words, if the relaxation time of the velocity distribution function of gaseous molecules is much shorter than the time scale of the change in the gaseous composition through the chemical reactions (e.g., Kitahara 1994).

\subsection{Elementary and overall reactions and their currents}

It is essential in the following discussion to distinguish an elementary reaction and an overall reaction. The elementary reaction is an actual reaction that is a step of a series of successive reactions (e.g.,
McQuarrie \& Simon 1997). In an elementary reaction, the products are formed directly from the reactants without passing intermediate compounds, thus the current of an elementary reaction is proportional to the product of the concentrations of the reactants (e.g., McQuarrie \& Simon 1997). This is a basic postulate of chemical kinetics, implying that the rate constant of an elementary reaction is independent of the concentrations.

In consequence, the net current of the reaction

$\mathcal{X}+\mathcal{B}_{1}+\cdots+\mathcal{B}_{n} \rightleftharpoons \mathcal{Y}+\mathcal{D}_{1}+\cdots+\mathcal{D}_{m}$

is expressed by

$J=k X B_{1} \cdots B_{n}-\alpha Y D_{1} \cdots D_{m}$

if the forward and reverse reactions are elementary reactions. Here, $X, B_{i}, Y, D_{i}$ represent the concentrations (which mean the number densities hereafter) of the molecular species $\mathcal{X}, \mathcal{B}_{i}, \mathcal{Y}$, and $\mathcal{D}_{i}$, and $k$ and $\alpha$ are the rate constants, which are independent of the concentrations.

An overall reaction is a net reaction summed over successive elementary reactions given, for example, by

$\mathcal{X}+\mathcal{B}_{1} \rightleftharpoons \mathcal{Z}_{1}+\mathcal{D}_{11}+\cdots$

$\mathcal{Z}_{1}+\mathcal{B}_{2} \rightleftharpoons \mathcal{Z}_{2}+\mathcal{D}_{21}+\cdots$

$\cdots$

$\mathcal{Z}_{n-1}+\mathcal{B}_{n} \rightleftharpoons \mathcal{Y}+\mathcal{D}_{n 1}+\cdots$

The overall reaction of a sequence of these elementary reactions is expressed by

$\mathcal{X}+\mathcal{B}_{1}+\cdots+\mathcal{B}_{n} \rightleftharpoons \mathcal{Y}+\mathcal{F}_{1}+\cdots$,

where $\mathcal{F}_{1}+\cdots=\mathcal{D}_{11}+\cdots+\mathcal{D}_{n 1}+\cdots$. Here we have put the stoichiometric coefficients of the species participating in the reactions to be unity without loss of generality. It should be pointed out that one should distinguish the overall reaction (7) from the elementary reaction (2) despite their apparent identity; the reaction (7) is not a reaction that occurs as an elementary process, but is a sum of the set of the elementary reactions (4) to (6).

In the steady state, the net currents are all identical for those reactions including the overall reaction (7). We present a principle relation on the steady current for the overall reaction (7) expressed by

$J=k_{\mathcal{X}} X B_{1} \cdots B_{n}-\alpha \mathcal{Y} Y F_{1} \cdots$,

where $k_{\mathcal{X}}$ and $\alpha \mathcal{y}$ are not independent of the concentrations but functions of the concentrations $B_{1}, \cdots, B_{n}$ and $F_{1}, \cdots$, respectively. This is verified as follows: in the reaction (7), the forward current must vanish if any one of the concentrations of the molecular species $\mathcal{X}, \mathcal{B}_{1}, \cdots, \mathcal{B}_{n}$ participating in the forward reaction is zero, because all of them are required for the forward reaction to proceed. The forward current must thus include a factor $X B_{1} \cdots B_{n}$. The same holds for the reverse current as well. In consequence, we have Eq. (8). The rate constants $k_{\mathcal{X}}$ and $\alpha_{\mathcal{Y}}$ 
are functions of the concentrations and remain finite in the tenuous limit where the concentrations tend to be zero.

The relation (8) states that the current of the overall reaction is of the same form as that of an elementary reaction but the rate constants are no longer independent of the concentrations. We call $k_{\mathcal{X}}$ and $\alpha \mathcal{y}$ that appear in the overall reaction the effective rate constants to distinguish from the rate constants in the elementary reactions. The effective rate constants depend on the rate constants of the elementary reactions as well as the concentrations of the molecular species $B_{1}, \cdots, B_{n}, F_{1}, \cdots$ participating in the overall reaction (7). It can be shown (see Appendix A) that the ratio of the effective rate constants $\alpha_{\mathcal{Y}}$ and $k_{\mathcal{X}}$ appearing in Eq. (8) is independent of the concentrations $B_{1}, \cdots, B_{n}$ and $F_{1}, \cdots$ in the overall reaction $(7)$. This fact plays a basic role in deriving the steady nucleation rate as discussed in Sect. 3 .

\subsection{Multiple paths}

In producing $\mathcal{Y}$ from $\mathcal{X}$ through successive reactions with $\mathcal{B}_{1}, \cdots, \mathcal{B}_{n}$, there are $n$ ! paths depending on the order of the reactions with $\mathcal{B}_{1}, \cdots, \mathcal{B}_{n}$ if $\mathcal{B}_{1}, \cdots, \mathcal{B}_{n}$ are different species with each other. The total current is given by the sum of the current $J_{i}$ given by the form (8) for each path $i$ :

$J=k X B_{1} \cdots B_{n}-\alpha Y F_{1} \cdots$

with

$k=\sum_{i} k_{i}, \quad \alpha=\sum_{i} \alpha_{i}=\sum_{i} \beta_{i} k_{i}$,

where the summation is taken over $n$ ! paths, and $\beta_{i} \equiv$ $\alpha_{i} / k_{i}$ is concentration-independent. Note that the "rate constants" of the total reaction are given by the sum of the effective rate constants for each path. The paths having the largest $k_{i}$ and $\alpha_{i}$ contribute most to the total current $J$.

In what follows, we discuss the current for one path for each successive reaction and omit the suffix $i$ from $J_{i}$.

\section{Formulation of the chemical nucleation rate}

\subsection{Definition of the equilibrium state}

We define an equilibrium state to be a state in which a vapor and a bulk condensate coexist in a closed system at a given temperature and pressure. The vapor consists of molecules and clusters of various sizes from which grains will form. The present definition of equilibrium is the same as that employed by Patzer et al. (1998) following Katz \& Wiedersich (1977), and does not mean a metastable supersaturated-state in the absence of a bulk condensate employed in the conventional nucleation theory (e.g., Feder et al. 1966; Abraham 1974).

The equilibrium state has the following important properties: 1) Both vapor and bulk condensate are of equal temperature, 2) the forward current equals the reverse current for any reaction of both elementary and overall reactions, thus $J=0$ for any reaction, and 3) chemical equilibrium is realized among various species and phases, implying that the sums of chemical potentials of the reactants and of the products are equal for any reaction involving various molecular species in the vapor and bulk condensate.

The equilibrium state is used as a reference state in dealing with actual states usually in a supersaturated state, in which nucleation occurs. The quantities in the equilibrium state are denoted by putting dot "." above their symbols hereafter, which we use instead of "०" used, for example, by Patzer et al. (1998) to avoid confusion with quantities at a standard pressure. The readers should not misunderstand that the dot indicates time derivative.

\subsection{Steady nucleation rate}

We shall deal with steady nucleation involving chemical reactions in which $n$ kinds of reactants $\mathcal{B}_{1}, \cdots, \mathcal{B}_{n}$ participate in the reaction. Furthermore, we do not assume in general the presence of monomers having the same composition as the grains and clusters whose size is larger than a certain size. The clusters with sizes larger than a certain size, for which we put $p$, will have the same composition as the grains of a macroscopic size, say, larger than $0.1 \mu \mathrm{m}$. We do not consider cluster-cluster reactions (Patzer et al. 1998) but deal with stepwise growth and dissociation of the clusters $\mathcal{A}_{i}$, i.e. $\mathcal{A}_{i} \rightleftharpoons \mathcal{A}_{i+1}$. The stepwise growth is realized if the concentrations of the reactants are large compared with those of the clusters. Furthermore, we restrict our discussion to the case that reactant molecules and clusters of various sizes are of equal temperature, i.e. in the LTE state in the sense of Patzer et al. (1998).

In the situation stated above, the clusters of size $i(>p)$ grow to the size $i+1$ through the reactions with $\mathcal{B}_{1}, \cdots, \mathcal{B}_{n}$. For $i \geq p$, the overall reaction for the formation of a cluster $\mathcal{A}_{i+1}$ from a cluster $\mathcal{A}_{i}$ is written as

$\mathcal{A}_{i}+\mathcal{B}_{1}+\cdots+\mathcal{B}_{n} \rightleftharpoons \mathcal{A}_{i+1}+\sum_{k} f_{k} \mathcal{F}_{k} \quad(i \geq p)$,

where $\mathcal{F}_{k}$ are the product species other than $i+1$-mers, $\mathcal{A}_{i+1}$, and $f_{k}$ are the stoichiometric coefficients. The elementary reactions to reach $p$-mers, $\mathcal{A}_{p}$, cannot be specified in general, but the overall reaction is written as

$p\left(\mathcal{B}_{1}+\cdots+\mathcal{B}_{n}\right) \rightleftharpoons \mathcal{A}_{p}+p \sum_{k} f_{k} \mathcal{F}_{k}$.

Here we have assumed that the reactants for the $\mathcal{A}_{p}$ formation are the same as those for $\mathcal{A}_{i}(i \geq p)$ formation. The net reaction for forming $\mathcal{A}_{i}$ from the reactant $\mathcal{B}_{1}, \cdots$, $\mathcal{B}_{n}$ is given by

$i\left(\mathcal{B}_{1}+\cdots+\mathcal{B}_{n}\right) \rightleftharpoons \mathcal{A}_{i}+i \sum_{k} f_{k} \mathcal{F}_{k}$

In the bulk limit of $i \rightarrow \infty$, the reaction (13) is expressed by

$\mathcal{B}_{1}+\cdots+\mathcal{B}_{n} \rightleftharpoons \mathcal{A}_{\mathrm{s}}+\sum_{k} f_{k} \mathcal{F}_{k}$ 
where $\mathcal{A}_{\mathrm{s}}$ represents a molecular unit of the bulk condensate.

Let us denote the concentrations (i.e. number densities) of $\mathcal{B}_{1}, \cdots, \mathcal{B}_{n}$ by $c_{1}, \cdots, c_{n}$, respectively, those of $\mathcal{F}_{k}$ by $c_{\mathcal{F}_{k}}$, and those of $\mathcal{A}_{i}$ by $c(i)$. From Eq. (8), the steady current, which equals the steady nucleation rate here, is expressed by

$$
J=k_{i} c_{1} \cdots c_{n} c(i)-\alpha_{i+1} \prod_{k} c_{\mathcal{F}_{k}}^{f_{k}} \cdot c(i+1)
$$

for the reaction (11), where $k_{i}$ and $\alpha_{i+1}$ are the effective forward and reverse rate constants, respectively. For the reaction (12), the steady nucleation rate is expressed by

$$
J=k_{1, p}\left(c_{1} \cdots c_{n}\right)^{p}-\alpha_{p} \prod_{k}\left(c_{\mathcal{F}_{k}}^{f_{k}}\right)^{p} \cdot c(p),
$$

where $k_{1, p}$ and $\alpha_{p}$ are the effective rate constants. Note that both $J$ given by Eqs. (15) and (16) are equal in the steady state.

Since $J=0$ in equilibrium, we can rewrite $J$ given by (16) and (15) with the use of the equilibrium concentrations as

$$
\begin{aligned}
J & =k_{1, p}\left(c_{1} \cdots c_{n}\right)^{p}\left[1-\frac{1}{S^{p}} \cdot \frac{c(p)}{\dot{c}(p)}\right] \\
& =k_{i} c_{1} \cdots c_{n} \dot{c}(i)\left[\frac{c(i)}{\dot{c}(i)}-\frac{1}{S} \cdot \frac{c(i+1)}{\dot{c}(i+1)}\right] \quad(i \geq p),
\end{aligned}
$$

where $\dot{c}(i)$, etc. are the equilibrium concentrations, and $S$ is defined by

$S=\frac{c_{1} \cdots c_{n}}{\dot{c}_{1} \cdots \dot{c}_{n}} \prod_{k}\left(\frac{\dot{c}_{\mathcal{F}_{k}}}{c_{\mathcal{F}_{k}}}\right)^{f_{k}}=\frac{P_{1} \cdots P_{n}}{\dot{P}_{1} \cdots \dot{P}_{n}} \prod_{k}\left(\frac{\dot{P}_{\mathcal{F}_{k}}}{P_{\mathcal{F}_{k}}}\right)^{f_{k}}$

where $P_{i}=c_{i} k_{\mathrm{B}} T$ and $\dot{P}_{i}=\dot{c}_{i} k_{\mathrm{B}} T$ with $k_{\mathrm{B}}$ being Boltzmann's constant. In deriving Eqs. (17) and (18), we have used the relations $\alpha_{p} / k_{1, p}=\dot{\alpha}_{p} / \dot{k}_{1, p}$ and $\alpha_{i+1} / k_{i}=$ $\dot{\alpha}_{i+1} / \dot{k}_{i}$ (see Sect. 2.1), where $\dot{k}_{i}, \dot{\alpha}_{i+1}$, etc. are the effective rate constants for the equilibrium concentrations. From the recurrence relations (17) and (18), $J$ is obtained by the conventional procedure (e.g., Katz \& Wiedersich 1977; Kozasa \& Hasegawa 1987; Gail \& Sedlmayr 1988; Patzer et al. 1998; Chigai et al. 1999). Namely, multiplying $1 / S^{i}$ on both sides of Eq. (18) divided by $k_{i} c_{1} \cdots c_{n} \dot{c}(i)$ successively for $i=p, p+1, p+2, \cdots$, summing over $i$, and adding Eq. (17) divided by $k_{1, p}\left(c_{1} \cdots c_{n}\right)^{p}$, we obtain

$$
\frac{1}{J}=\frac{1}{c_{1} \cdots c_{n}}\left[\frac{1}{k_{1, p}\left(c_{1} \cdots c_{n}\right)^{p-1}}+\sum_{i=p}^{\infty} \frac{1}{k_{i} S^{i} \dot{c}(i)}\right],
$$

where we have used

$$
\lim _{i \rightarrow \infty} \frac{1}{S^{i+1}} \cdot \frac{c(i+1)}{\dot{c}(i+1)}=0
$$

for $S>1$. The proof of Eq. (21) is given in Appendix B.
From the equilibrium condition of the reaction (13) of $i$-mer formation, we have

$$
i \sum_{j=1}^{n} \dot{\mu}_{j}=\dot{\mu}(i)+i \sum_{k} f_{k} \dot{\mu}_{\mathcal{F}_{k}}
$$

where the chemical potentials per molecule of $\mathcal{B}_{j}$ in equilibrium are denoted by $\dot{\mu}_{j}$, those of $\mathcal{A}_{i}$ by $\dot{\mu}(i)$, and those of $\mathcal{F}_{k}$ by $\dot{\mu}_{\mathcal{F}_{k}}$. For a gaseous species $a$ of partial pressure $\dot{P}_{a}$ and temperature $T$, the chemical potential per molecule is expressed (e.g., Landau \& Lifschitz 1980) by

$\dot{\mu}_{a}=\chi_{a}+k_{\mathrm{B}} T \ln \frac{\dot{P}_{a}}{P_{0}}=\chi_{a}+k_{\mathrm{B}} T \ln \frac{\dot{c_{a}} k_{\mathrm{B}} T}{P_{0}}$

if the vapor can be regarded to be an ideal gas, where $\chi_{a}$ is the chemical potential per molecule at a standard pressure $P_{0}$. Using this expression for the chemical potentials in Eq. (22), we obtain the equilibrium concentrations $\dot{c}(i)$ of $\mathcal{A}_{i}$ as

$$
\begin{aligned}
\dot{c}(i) & =\frac{P_{0}}{k_{\mathrm{B}} T} \mathrm{e}^{-g^{\circ}(i)}\left[\frac{\dot{P}_{1} \cdots \dot{P}_{n}}{P_{0}^{n}} \prod_{k}\left(\frac{P_{0}}{\dot{P}_{\mathcal{F}_{k}}}\right)^{f_{k}}\right]^{i} \\
& =\frac{P_{0}}{k_{\mathrm{B}} T} \mathrm{e}^{-g^{\circ}(i)} \frac{1}{S^{i}}\left[\frac{P_{1} \cdots P_{n}}{P_{0}^{n}} \prod_{k}\left(\frac{P_{0}}{P_{\mathcal{F}_{k}}}\right)^{f_{k}}\right]^{i}
\end{aligned}
$$

with

$g^{\circ}(i)=\frac{1}{k_{\mathrm{B}} T}\left[\chi(i)+i \sum_{k} f_{k} \chi_{\mathcal{F}_{k}}-i \sum_{j=1}^{n} \chi_{j}\right]$

$(i \geq p)$.

Substitution of $\dot{c}(i)$ given by Eq. (23) into Eq. (20) leads to the steady nucleation rate $J$ expressed in terms of the given quantities as

$$
\begin{aligned}
& \frac{1}{J}=\frac{1}{c_{1} \cdots c_{n}}\left[\frac{1}{k_{1, p}\left(c_{1} \cdots c_{n}\right)^{p-1}}\right. \\
& \left.+\sum_{i=p}^{\infty}\left\{k_{i} \frac{P_{0}}{k_{\mathrm{B}} T}\left[\frac{P_{1} \cdots P_{n}}{P_{0}^{n}} \prod_{k}\left(\frac{P_{0}}{P_{\mathcal{F}_{k}}}\right)^{f_{k}}\right]^{i} \mathrm{e}^{-g^{\circ}(i)}\right\}^{-1}\right] .
\end{aligned}
$$

\subsection{One-component system}

To examine the steady chemical nucleation rate given by Eq. (25), let us discuss the simplest case, i.e. a onecomponent system in this subsection. The reaction for $i$ mer formation is given by

$\mathcal{A}_{i}+\mathcal{A} \rightleftharpoons \mathcal{A}_{i+1} \quad(i \geq 1)$.

Comparing this reaction with the reaction (11), we see $n=p=1, f_{k}=0, c_{1}=c(1)$ and $\mathcal{B}_{1}=\mathcal{A}$, indicating that the reactant $\mathcal{B}_{1}$ acts as a monomer $\mathcal{A}$ in this case; the reaction (12) reduces to an identical reaction, and needs 
not to be taken into account. In addition, $S$ defined by Eq. (19) reduces to

$S=P_{1} / \dot{P}_{1}$,

which equals a supersaturation ratio in a one-component system. For a one-component system, the rate constant is expressed by $k_{i}=\left\langle\sigma_{i} v\right\rangle$ with using cross section $\sigma_{i}$ of an $i$-mer for collisional sticking of monomers, and velocity $v$ of monomers relative to the $i$-mer, where $\langle\cdots\rangle$ indicates an average over a Maxwellian velocity distribution. For ideal gases, $k_{i}$ is given by $k_{i}=\alpha s_{i} \sqrt{k_{\mathrm{B}} T / 2 \pi m}$, where $\alpha$ is the condensation coefficient, $s_{i}$ is the surface area of an $i$-mer, and $m$ is the reduced mass of a monomer and an $i$-mer, almost equal to a monomer mass if $i \gg 1$. It is easily confirmed that, for $\alpha$ being independent of the monomer concentration, Eq. (20) for $n=1$ reduces to Eq. (9) of Katz \& Wiedersich (1977), who derived the steady nucleation rate for a one-component system by defining the equilibrium state to be a saturated state as stated in Sect. 3.1.

Ignoring the first term on the RHS of Eq. (25) resulting from the reaction (12), we have

$$
\begin{aligned}
\frac{1}{J} & =\sum_{i=1}^{\infty}\left\{k_{i} c_{1} \frac{P_{0}}{k_{\mathrm{B}} T} \frac{P_{1}}{P_{0}}\left(\frac{P_{1}}{P_{0}}\right)^{i-1} \mathrm{e}^{-g^{\circ}(i)}\right\}^{-1} \\
& =\sum_{i=1}^{\infty}\left\{k_{i} c_{1}^{2} \mathrm{e}^{-g(i)}\right\}^{-1}
\end{aligned}
$$

with

$$
\begin{aligned}
g^{\circ}(i) & =\left[\chi(i)-i \chi_{1}\right] / k_{\mathrm{B}} T, \\
g(i) & =\left[\chi(i)-i \chi_{1}\right] / k_{\mathrm{B}} T-(i-1) \ln \left(P_{1} / P_{0}\right) .
\end{aligned}
$$

From a comparison of Eq. (28) with the conventional nucleation rate for a one-component system (e.g., Feder et al. 1966; Abraham 1974), we see that $k_{\mathrm{B}} T g(i)$ is the minimum work needed to form an $i$-mer $\mathcal{A}_{i}$ from monomers $\mathcal{A}$ (Landau \& Lifschitz 1980); the minimum work equals the Gibbs free energy change for formation of $\mathcal{A}_{i}$ from $\mathcal{A}$ if the clusters and the vapor in a closed system are of equal temperature and pressure (Landau \& Lifschitz 1980).

To obtain a more familiar expression of $g(i)$, let us introduce the equilibrium state as a reference state, and consider chemical equilibrium between monomers and a bulk condensate (cf. Eq. (14)):

$\mathcal{A} \rightleftharpoons \mathcal{A}_{\mathrm{s}}$

The equilibrium condition of this reaction, $\dot{\mu}_{1}=\mu_{\mathrm{s}}$, is expressed by

$\chi_{1}+k_{\mathrm{B}} T \ln \frac{\dot{P}_{1}}{P_{0}}=\mu_{\mathrm{s}}$

where $\mu_{\mathrm{s}}$ is the chemical potential per $\mathcal{A}_{\mathrm{s}}$, and is independent of pressures concerned. From Eqs. (27) and (32), the supersaturation ratio $S$ is expressed by

$\ln S=\ln \frac{P_{1}}{P_{0}}-\frac{\mu_{\mathrm{s}}-\chi_{1}}{k_{\mathrm{B}} T}=\frac{\mu_{1}-\mu_{\mathrm{s}}}{k_{\mathrm{B}} T}$, where $\mu_{1}$ is the chemical potential of a monomer $\mathcal{A}$ defined for a supersaturated (i.e. nonequilibrium) state (e.g., Kitahara 1994), and is given by

$\mu_{1}=\chi_{1}+k_{\mathrm{B}} T \ln \left(P_{1} / P_{0}\right)$.

As is expected, $\ln S=0$ in equilibrium from Eq. (32).

Rewriting $g(i)$ given by Eq. (30) with the use of $\mu_{\mathrm{s}}$ and Eq. (33), we obtain

$$
\begin{aligned}
g(i)= & -(i-1)\left(\ln \frac{P_{1}}{P_{0}}-\frac{\mu_{\mathrm{s}}-\chi_{1}}{k_{\mathrm{B}} T}\right) \\
& +\frac{\chi(i)-\chi_{1}-(i-1) \mu_{\mathrm{s}}}{k_{\mathrm{B}} T} \\
= & -(i-1) \ln S+\frac{\chi(i)-\chi_{1}-(i-1) \mu_{\mathrm{s}}}{k_{\mathrm{B}} T} .
\end{aligned}
$$

For $i \gg 1, g(i)$ is approximated to be:

$g(i) \simeq-i \ln S+\frac{\chi(i)-i \mu_{\mathrm{s}}}{k_{\mathrm{B}} T}$,

where the first and second terms represent the volume and the surface energies, respectively.

\subsection{Another expression of the steady chemical nucleation rate}

Rewriting Eq. (25) in the similar manner as in Eq. (28), we have

$$
\begin{aligned}
& \frac{1}{J}=\frac{1}{c_{1} \cdots c_{n}}\left[\frac{1}{k_{1, p}\left(c_{1} \cdots c_{n}\right)^{p-1}}\right. \\
& \left.+\sum_{i=p}^{\infty}\left\{k_{i} \frac{P_{0}}{k_{\mathrm{B}} T} \frac{P_{1} \cdots P_{n}}{P_{0}^{n}} \prod_{k}\left(\frac{P_{0}}{P_{\mathcal{F}_{k}}}\right)^{f_{k}} \mathrm{e}^{-g(i)}\right\}^{-1}\right],
\end{aligned}
$$

where $g(i)$ is defined by

$$
\begin{aligned}
g(i)= & g^{\circ}(i)-(i-1) \ln \left[\frac{P_{1} \cdots P_{n}}{P_{0}^{n}} \prod_{k}\left(\frac{P_{0}}{P_{\mathcal{F}_{k}}}\right)^{f_{k}}\right] \\
= & \frac{1}{k_{\mathrm{B}} T}\left\{-(i-1)\left[\sum_{j=1}^{n} \mu_{j}-\sum_{k} f_{k} \mu_{\mathcal{F}_{k}}\right]\right. \\
& \left.+\left[\chi(i)+\sum_{k} f_{k} \chi_{\mathcal{F}_{k}}-\sum_{j=1}^{n} \chi_{j}\right]\right\}
\end{aligned}
$$

where

$\mu_{a}=\chi_{a}+k_{\mathrm{B}} T \ln \frac{P_{a}}{P_{0}} \quad\left(a\right.$ is $\mathcal{B}_{j}$ or $\left.\mathcal{F}_{k}\right)$

is the chemical potential per molecule of gaseous species $a$ at partial pressure $P_{a}$ (cf. Eq. (34)). Using the chemical potential $\mu_{\mathrm{s}}$ per $\mathcal{A}_{\mathrm{s}}$ of the bulk condensate, $g(i)$ is expressed by

$$
\begin{aligned}
g(i)= & \frac{1}{k_{\mathrm{B}} T}\left[-(i-1)\left(\sum_{j=1}^{n} \mu_{j}-\mu_{\mathrm{s}}-\sum_{k} f_{k} \mu_{\mathcal{F}_{k}}\right)\right. \\
& \left.+\left(\chi(i)-(i-1) \mu_{\mathrm{s}}+\sum_{k} f_{k} \chi_{\mathcal{F}_{k}}-\sum_{j=1}^{n} \chi_{j}\right)\right]
\end{aligned}
$$


which reduces to the minimum work $g(i)$ given by Eq. (35) when $n=1$ and $f_{k}=0$, implying that $g(i)$ represents the minimum work to form an $i$-mer $\mathcal{A}_{i}$ from reactant molecules $\mathcal{B}_{1}, \cdots, \mathcal{B}_{n}$ for $i \gg 1$.

\subsection{Supersaturation ratio}

The equilibrium condition of the reaction (14) is expressed by

$\mu_{\mathrm{s}}+\sum_{k} f_{k} \dot{\mu}_{\mathcal{F}_{k}}-\sum_{j=1}^{n} \dot{\mu}_{j}=0$.

Subtracting the LHS of Eq. (42) divided by $k_{\mathrm{B}} T$ from $\ln S$ with $S$ defined by Eq. (19), we have

$\ln S=\frac{1}{k_{\mathrm{B}} T}\left(\sum_{j=1}^{n} \mu_{j}-\mu_{\mathrm{s}}-\sum_{k} f_{k} \mu_{\mathcal{F}_{k}}\right)$,

which reproduces the supersaturation ratio given by Eq. (33) for a one-component system by putting $n=1$ and $f_{k}=0$, so $S$ given by Eq. (43) is regarded to be a natural extension of the supersaturation ratio to a manycomponent system (Kozasa \& Hasegawa 1987).

In terms of the supersaturation ratio $S$ newly defined by Eq. (43), $g(i)$ given by Eq. (41) is expressed as

$$
\begin{aligned}
& g(i)=-(i-1) \ln S \\
& +\frac{1}{k_{\mathrm{B}} T}\left\{\chi(i)-(i-1) \mu_{\mathrm{s}}+\sum_{k} f_{k} \chi_{\mathcal{F}_{k}}-\sum_{j=1}^{n} \chi_{j}\right\} .
\end{aligned}
$$

If $i \gg 1, g(i)$ may be approximated leading to the same form as given by Eq. (37) for the one-component system. The first term on the RHS represents the volume energy of an $i$-mer as is in the one-component system. The second term, which is proportional to $\chi(i)-i \mu_{\mathrm{s}}$ if $i \gg 1$, indicates the energy difference arising from the free surface, when an $i$-mer from the bulk condensate is moved into the vapor. Thus, $\chi(i)-i \mu_{\mathrm{s}} \propto i^{2 / 3}$ if $i \gg 1$ for spherical $i$-mers, and represents the surface energy. In consequence, $g(i)$ given by Eq. (44) is approximated if $i \gg 1$ to be:

$g(i) \simeq-i \ln S+\theta_{\infty} i^{2 / 3}$

with

$\theta_{\infty}=\frac{4 \pi \sigma a_{0}^{2}}{k_{\mathrm{B}} T}$

where $\sigma$ is the surface tension of the bulk condensate, and $a_{0}$ is the equivalent radius of a molecular unit of the bulk condensate. It should be noted that $g(i)$ given by Eq. (44) attains a maximum at $i=i_{*}$ given by

$i_{*} \simeq\left(\frac{2 \theta_{\infty}}{3 \ln S}\right)^{3}$

if $i_{*} \gg 1$.

\subsection{Transition probabilities}

The steady nucleation rate (15) is expressed as

$J=p_{i} c(i)-r_{i+1} c(i+1)$

with

$p_{i}=k_{i} c_{1} \cdots c_{n} \quad$ and $\quad r_{i+1}=\alpha_{i+1} \prod_{k} c_{\mathcal{F}_{k}}^{f_{k}}$.

In a one-component system, $p_{i}$ is the growth rate, i.e. the number of monomers sticking onto an $i$-mer per unit time, and $r_{i+1}$ is the evaporation rate, i.e. the number of monomers evaporating from $i+1$-mer per unit time. In an $n$-component system, $p_{i}$ indicates the probability of transition from $\mathcal{A}_{i}$ to $\mathcal{A}_{i+1}$ per unit time, and $r_{i+1}$ the probability of transition from $\mathcal{A}_{i+1}$ to $\mathcal{A}_{i}$ per unit time.

The ratio of the transition probabilities is given from Eq. (49) by

$\frac{r_{i+1}}{p_{i}}=\frac{1}{S} \cdot \frac{\alpha_{i+1} \prod_{k} \dot{c}_{\mathcal{F}_{k}}^{f_{k}}}{k_{i} \dot{c}_{1} \cdots \dot{c}_{n}}$

with the use of Eq. (19). Since $J=0$ in equilibrium, we have

$\frac{\dot{r}_{i+1}}{\dot{p}_{i}}=\frac{\dot{\alpha}_{i+1} \prod_{k} \dot{c}_{\mathcal{F}_{k}}^{f_{k}}}{\dot{k}_{i} \dot{c}_{1} \cdots \dot{c}_{n}}=\frac{\dot{c}(i)}{\dot{c}(i+1)}$

from Eqs. (49) and (48). Equations (50) and (51) yield

$\frac{r_{i+1}}{p_{i}}=\frac{1}{S} \cdot \frac{\dot{c}(i)}{\dot{c}(i+1)}$,

where we have put $\left(\alpha_{i+1} / k_{i}\right) /\left(\dot{\alpha}_{i+1} / \dot{k}_{i}\right)=1$ (see Sect. 2.1). The equilibrium concentrations $\dot{c}(i)$ and $\dot{c}(i+1)$ are given by Eq. (23), which leads with the use of Eq. (39) to

$\frac{\dot{c}(i)}{\dot{c}(i+1)}=S \exp [g(i+1)-g(i)]$.

Thus, the ratio of the transition probabilities (50) is given by

$\frac{r_{i+1}}{p_{i}}=\exp [g(i+1)-g(i)]$.

The relation (54) is of exactly the same form as the ratio of the evaporation rate to the growth rate for a onecomponent system, and indicates that $r_{i+1} / p_{i}>1$ for cluster sizes $i$ with $g^{\prime}(i)>0$ and $r_{i+1} / p_{i}<1$ for sizes $i$ with $g^{\prime}(i)<0$. The clusters for which the forward transition probability equals the reverse current are the critical clusters, whose size $i_{*}$ is given by the size where $g(i)$ attains a maximum $\left(g^{\prime}\left(i_{*}\right)=0\right)$, i.e. by a solution to

$\chi^{\prime}\left(i_{*}\right)=\mu_{\mathrm{s}}+k_{\mathrm{B}} T \ln S$

from Eq. (44). These results confirm that $g(i)$ given by Eq. (44) has the properties required for the minimum work needed to form an $i$-mer of sizes $i \lesssim i_{*}$ as well as of $i \gg 1$ as discussed in Sect. 3.4. 


\section{Steady chemical nucleation rate in the presence of monomers}

The reaction (1) that Girshick (1997) dealt with is expressed as

$\mathcal{A}_{i}+\mathcal{B}_{1} \rightarrow \mathcal{A}_{i+1}+\mathcal{F}_{1} \quad(i \geq 1)$.

A characteristic of this reaction is the presence of monomers $\mathcal{A}_{1}$ from which a sequence of the reactions starts. Here we deal with an extended type of such reactions

$\mathcal{A}_{i}+\mathcal{B}_{1}+\cdots+\mathcal{B}_{n} \rightarrow \mathcal{A}_{i+1}+\sum_{k} f_{k} \mathcal{F}_{k} \quad(i \geq 1)$,

which is an overall reaction, and is comparable to a set of the reactions of (11) and (12) in the absence of monomers, where a monomer $\mathcal{A}_{1}$ nominally corresponds to

$\mathcal{A}_{1} \equiv \mathcal{B}_{1}+\cdots+\mathcal{B}_{n}-\sum_{k} f_{k} \mathcal{F}_{k}$

from Eq. (12) for $p=1$. The net reaction for forming $\mathcal{A}_{i}$ from $\mathcal{A}_{1}$ and $\mathcal{B}_{1}, \cdots, \mathcal{B}_{n}$ is expressed by

$$
\mathcal{A}_{1}+(i-1)\left(\mathcal{B}_{1}+\cdots+\mathcal{B}_{n}\right) \rightarrow \mathcal{A}_{i}+(i-1) \sum_{k} f_{k} \mathcal{F}_{k}
$$$$
(i \geq 1)
$$

which is comparable to the reaction (13).

The steady nucleation rate for the reaction (57) is given by

$$
\begin{aligned}
J & =k_{i} c(i) c_{1} \cdots c_{n}-\alpha_{i+1} c(i+1) \prod_{k} c_{\mathcal{F}_{k}}^{f_{k}} \\
& =k_{i} \dot{c}(i) c_{1} \cdots c_{n}\left[\frac{c(i)}{\dot{c}(i)}-\frac{1}{S} \cdot \frac{c(i+1)}{\dot{c}(i+1)}\right],
\end{aligned}
$$

which holds for $i \geq 1$ because of the presence of monomers. Similar calculations as in Sect. 3 yield

$$
\begin{aligned}
& \frac{1}{J}=\frac{1}{c(1) c_{1} \cdots c_{n}} \\
& \times \sum_{i=1}^{\infty}\left\{k_{i}\left[\frac{P_{1} \cdots P_{n}}{P_{0}^{n}} \prod_{k}\left(\frac{P_{0}}{P_{\mathcal{F}_{k}}}\right)^{f_{k}}\right]^{i-1} \mathrm{e}^{-g^{\circ}(i)}\right\}^{-1},
\end{aligned}
$$

where

$$
\begin{aligned}
g^{\circ}(i)= & \frac{1}{k_{\mathrm{B}} T}\left[\chi(i)+(i-1) \sum_{k} f_{k} \chi_{\mathcal{F}_{k}}-\chi(1)\right. \\
& \left.-(i-1) \sum_{j=1}^{n} \chi_{j}\right] .
\end{aligned}
$$

Note that $g^{\circ}(i)$ given by Eq. (62) is the same as that defined by Eq. (24) in the absence of monomers if one makes a formal identification given by Eq. (66) below. In terms of the minimum work for $i$-mer formation given by

$$
\begin{aligned}
g(i) & =g^{\circ}(i)-(i-1) \ln \left[\frac{P_{1} \cdots P_{n}}{P_{0}^{n}} \prod_{k}\left(\frac{P_{0}}{P_{\mathcal{F}_{k}}}\right)^{f_{k}}\right] \\
& =-(i-1) \ln S+\left\{\chi(i)-\chi(1)-(i-1) \mu_{\mathrm{s}}\right\} / k_{\mathrm{B}} T
\end{aligned}
$$

with $S$ given by Eq. (43), the steady nucleation rate $J$ is expressed by

$\frac{1}{J}=\frac{1}{c(1) c_{1} \cdots c_{n}} \sum_{i=1}^{\infty}\left\{k_{i} \mathrm{e}^{-g(i)}\right\}^{-1}$,

which is comparable to Eq. (38).

It is worth pointing out that the nucleation rate (64) can also be derived from Eq. (38) by the following purely formal procedure, which may help understanding the difference between the reaction (57) and a set of the reactions (11) and (12) with putting $p=1$ formally. Writing the reaction (13) as

$$
\begin{aligned}
& \underbrace{\left[\mathcal{B}_{1}+\cdots+\mathcal{B}_{n}-\sum_{k} f_{k} \mathcal{F}_{k}\right]}_{\mathcal{A}_{1}}+(i-1)\left(\mathcal{B}_{1}+\cdots+\mathcal{B}_{n}\right) \\
& \rightarrow \mathcal{A}_{i}+(i-1) \sum_{k} f_{k} \mathcal{F}_{k},
\end{aligned}
$$

and identifying a monomer $\mathcal{A}_{1}$ to that given by Eq. (58), then one can put formally

$$
\begin{aligned}
& \mu(1) \equiv \mu_{1}+\cdots+\mu_{n}-\sum_{k} f_{k} \mu_{\mathcal{F}_{k}} \\
& \chi(1) \equiv \chi_{1}+\cdots+\chi_{n}-\sum_{k} f_{k} \chi_{\mathcal{F}_{k}} .
\end{aligned}
$$

Applying the relation $\mu-\chi=k_{\mathrm{B}} T \ln \left(P / P_{0}\right)$ for an ideal gas formally, one obtains

$\frac{P_{0}}{k_{\mathrm{B}} T} \frac{P_{1} \cdots P_{n}}{P_{0}^{n}} \prod_{k}\left(\frac{P_{0}}{P_{\mathcal{F}_{k}}}\right)^{f_{k}}=c(1)$

by taking difference of both sides of Eqs. (65) and (66). The minimum work $g(i)$ given by Eq. (41) turns out to be Eq. (63) because of Eq. (66), thus the nucleation rate given by Eq. (38) without the first term of the r.h.s to be Eq. (64) from Eq. (67).

It is easily confirmed that the nucleation rate given by Eq. (61) reduces to that for a one-component system given by Eq. (28) by putting $n=1, f_{k}=0$ for all $k$ and $c(1)=c_{1}$.

Girshick's reaction (56) corresponds to the case

$n=1, \quad f_{1}=1, \quad f_{k}=0 \quad(k \geq 2)$

in the reaction (57). In this case, Eq. (61) with Eq. (62) reduces to

$\frac{1}{J}=\frac{1}{c(1) c_{1}} \sum_{i=1}^{\infty}\left\{k_{i}\left(\frac{P_{1}}{P_{\mathcal{F}_{1}}}\right)^{i-1} \mathrm{e}^{-g^{\circ}(i)}\right\}^{-1}$

with

$g^{\circ}(i)=\frac{1}{k_{\mathrm{B}} T}\left[\chi(i)+(i-1) \chi_{\mathcal{F}_{1}}-\chi(1)-(i-1) \chi_{1}\right]$,

which reproduces Eq. (18) for $M \rightarrow \infty$ in Girshick (1997). 


\section{Approximate formula of the chemical nucleation rate}

In the evaluation of the nucleation rate $J$ given by Eq. (38), we consider a situation that the formation of $p$-mer is so fast that the first term in the r.h.s of Eq. (38), which term is relevant to the $p$-mer formation, can be ignored. If it is not the case, a bottleneck of condensation of grains occurs before the clusters grow to $p$-mers, and the molecular processes of the $p$-mer formation become a problem to discuss, which is not a purpose of this paper.

In the situation stated above, we derive an approximate expression of the nucleation rate $J$. Replacing the summation in $J$ given by Eq. (38) by an integral, we have

$\sum_{i=p}^{\infty}\left\{k_{i} \mathrm{e}^{-g(i)}\right\}^{-1} \simeq \int_{p}^{\infty} \mathrm{d} i \frac{\mathrm{e}^{g(i)}}{k_{i}}$

When the minimum work $g(i)$ given by Eq. (44) has a maximum at $i=i_{*}$, a rate-determining step of nucleation is growth of the clusters with sizes around $i=i_{*}$. If $i_{*} \gg p$ and $k_{i}$ is a slowly varying function of $i$, the integral can be evaluated by the saddle point method with expanding $g(i)$ around $i=i_{*}$ as

$g(i) \simeq g\left(i_{*}\right)+\frac{g^{\prime \prime}\left(i_{*}\right)}{2}\left(i-i_{*}\right)^{2}$

and extending the lower limit of the integral to $-\infty$. The result is given by

$\sum_{i=p}^{\infty}\left\{k_{i} \mathrm{e}^{-g(i)}\right\}^{-1} \simeq \frac{\mathrm{e}^{g\left(i_{*}\right)}}{Z k_{i^{*}}}$,

where $Z=\sqrt{-g^{\prime \prime}\left(i_{*}\right) / 2 \pi}$ is the Zeldovich factor, which is a correction factor if the summation is represented by the maximum term at $i=i_{*}$. Thus, the nucleation rate $J$ given by Eq. (38) is approximated to be:

$J \simeq Z k_{i_{*}} c_{1} \cdots c_{n} \frac{P_{0}}{k_{\mathrm{B}} T} \frac{P_{1} \cdots P_{n}}{P_{0}^{n}} \prod_{k}\left(\frac{P_{0}}{P_{\mathcal{F}_{k}}}\right)^{f_{k}} \mathrm{e}^{-g\left(i_{*}\right)}$,

which is a good approximation if $-g^{\prime \prime}\left(i_{*}\right) \gg 1$. The size $i_{*}$ of a critical nucleus is given by a solution of Eq. (55), and is approximated by Eq. (47) if $i_{*} \gg 1$, while the Zeldovich factor is expressed by

$Z=\sqrt{\frac{-\chi^{\prime \prime}\left(i_{*}\right)}{2 \pi k_{\mathrm{B}} T}}$

from Eq. (44).

\section{Key species}

In dealing with nucleation accompanied with chemical reactions, Kozasa \& Hasegawa (1987) assumed that the nucleation rate was controlled by two-body collisions between the clusters and the molecules of a key species, which was defined as the species of the least collision frequencies among the reactants. Employing the key species concept, Chigai et al. (1999) have shown that the rate of steady chemical nucleation is expressed by a factor $\Pi$ times the steady nucleation rate for a one-component vapor ignoring the species other than the key species, where $\Pi$ is a function of partial pressures of the reactants and gaseous products other than the key species. If we denote the key species by $\mathcal{B}_{1}$, the nucleation rate of Chigai et al. (1999) is expressed by

$J=Z \Pi c_{1}^{2}\left\langle\sigma_{i_{*}} v\right\rangle \mathrm{e}^{-g\left(i_{*}\right)}$,

where

$\Pi=\frac{P_{2} \cdots P_{n}}{P_{0}{ }^{n-1}} \prod_{k}\left(\frac{P_{0}}{P_{\mathcal{F}_{k}}}\right)^{f_{k}}$,

and $\left\langle\sigma_{i_{*}} v\right\rangle$ is the rate constant for the reaction between a critical nucleus and a molecule of the key species, which is expressed by the product of the reaction cross section $\sigma_{i_{*}}$ of the critical nucleus and their relative velocity $v$ averaged over a Maxwellian velocity distribution.

Separating the quantities concerning $\mathcal{B}_{1}$, we can express the nucleation rate given by Eq. (38) as

$$
\begin{aligned}
\frac{1}{J} & =\frac{1}{k_{1, p}\left(c_{1} \cdots c_{n}\right)^{p}}+\frac{1}{\Pi c_{1}^{2}} \sum_{i=p}^{\infty}\left\{k_{i}^{\prime} \mathrm{e}^{-g(i)}\right\}^{-1} \\
& \simeq\left(Z \Pi c_{1}^{2} k_{i_{*}}^{\prime} \mathrm{e}^{-g\left(i_{*}\right)}\right)^{-1}
\end{aligned}
$$

where

$k_{i}^{\prime}=k_{i} c_{2} \cdots c_{n}$.

A comparison between Eqs. (72) and (74) indicates that the key species assumption corresponds to the replacement

$k_{i}^{\prime} \rightarrow\left\langle\sigma_{i} v\right\rangle$.

Let us examine under which conditions the concept of a key species is valid. We assume that a sequence of the reactions to form $\mathcal{A}_{i+1}$ from $\mathcal{A}_{i}$ starts from a reactive collision of a key species $\mathcal{B}_{1}$ onto $\mathcal{A}_{i}$ to form an intermediate compound $\mathcal{Z}_{i 1}$ such as

$\mathcal{A}_{i}+\mathcal{B}_{1} \rightleftharpoons \mathcal{Z}_{i 1}+\mathcal{D}_{11}+\mathcal{D}_{12}+\cdots$

In what follows, we consider the case of $n=2$, in which elementary reactions to form $\mathcal{A}_{i+1}$ from $\mathcal{A}_{i}$ are written as

$\mathcal{A}_{i}+\mathcal{B}_{1} \rightleftharpoons \mathcal{Z}_{i 1}+\mathcal{D}_{11}+\mathcal{D}_{12}+\cdots$

$\mathcal{Z}_{i 1}+\mathcal{B}_{2} \rightleftharpoons \mathcal{A}_{i+1}+\mathcal{D}_{21}+\mathcal{D}_{22}+\cdots$

The overall reaction of a sequence of the reactions (77) and (78) is given by

$\mathcal{A}_{i}+\mathcal{B}_{1}+\mathcal{B}_{2} \rightleftharpoons \mathcal{A}_{i+1}+\mathcal{D}_{11}+\cdots+\mathcal{D}_{21}+\cdots$

Expressing the steady currents of the elementary reactions by

$$
\begin{aligned}
J & =k_{i 1} c(i) c_{1}-\alpha_{i+1,1} c_{\mathcal{Z}_{i 1}} c_{\mathcal{D}_{11}} \cdots \\
& =k_{i 2} c_{\mathcal{Z}_{i 1}} c_{2}-\alpha_{i+1,2} c(i+1) c_{\mathcal{D}_{21}} \cdots
\end{aligned}
$$


we obtain the steady current of the overall reaction as

$J=k_{i} c(i) c_{1} c_{2}-\alpha_{i+1} c(i+1) c_{\mathcal{D}_{11}} \cdots c_{\mathcal{D}_{21}} \cdots$

with the effective rate constants given by

$$
\begin{aligned}
k_{i} & =\frac{k_{i 1} k_{i 2}}{k_{i 2} c_{2}+\alpha_{i+1,1} \prod_{j} c_{\mathcal{D}_{1 j}}}, \\
\alpha_{i+1} & =\frac{\alpha_{i+1,1} \alpha_{i+1,2}}{k_{i 2} c_{2}+\alpha_{i+1,1} \prod_{j} c_{\mathcal{D}_{1 j}}} .
\end{aligned}
$$

Note that the first term $k_{i 2} c_{2}$ in the denominators represents the rate of the forward reaction of (78), whereas the second term $\alpha_{i+1,1} c_{\mathcal{D}_{11}} c_{\mathcal{D}_{12}} \cdots$ represents the rate of the reverse reaction of $(77)$. If the relation

$\alpha_{i+1,1} c_{\mathcal{D}_{11}} c_{\mathcal{D}_{12}} \cdots \ll k_{i 2} c_{2}$,

holds, then $k_{i}^{\prime}=k_{i} c_{2}$ defined by Eq. (75) for $n=2$ may be approximated to be:

$k_{i}^{\prime} \simeq k_{i 1}=\left\langle\sigma_{i} v\right\rangle$,

and the replacement of (76) is justified. The condition (84) implies that the key-species approximation is valid if the reactive collisions of $\mathcal{B}_{2}$ onto the intermediate compounds $\mathcal{Z}_{i 1}$ to form $\mathcal{A}_{i+1}$ occur faster than the dissociation of $\mathcal{Z}_{i 1}$. It is plausible that the condition (84) is met in laboratory experiments of condensation in an inert gas, since the concentrations of $\mathcal{D}_{11}, \mathcal{D}_{12}, \cdots$, which are produced by the reactions of the key species $\mathcal{B}_{1}$ with $\mathcal{A}_{i}$, will satisfy the relation

$c_{\mathcal{D}_{11}}, c_{\mathcal{D}_{12}}, \cdots \lesssim c_{1} \ll c_{2}$

if $c_{1} \ll c_{2}$. When there are originally abundant product species $\mathcal{D}_{11}, \cdots$ in the condensation environment such as $\mathrm{H}_{2}$ in the carbon grain formation from $\mathrm{C}_{2} \mathrm{H}_{2}$ in C-rich AGB stars, on the other hand, the rate constant $\alpha_{i+1,1}$ must be low enough to compensate high concentrations of the product species.

The situation is essentially unchanged for $n>2$ as well. Formation of $\mathcal{A}_{i+1}$ from $\mathcal{A}_{i}$ proceeds through 1) initiation of the sequential reactions by formation of an intermediate compound $\mathcal{Z}_{i 1}$ through reactive collisions of the key species $\mathcal{B}_{1}$ with an $i$-mer $\mathcal{A}_{i}$, and 2) a subsequent series of the reactions forming $\mathcal{Z}_{i k}$ through reactive collisions of $\mathcal{B}_{k}(k=2, \cdots, n)$ with intermediate compounds $\mathcal{Z}_{i, k-1}$. The key-species approximation is valid if the forward reaction for forming $\mathcal{Z}_{i k}$ is faster than its dissociation in each step of the reactions.

To conclude this section, it should be pointed out that the key-species approximation provides one of the effective ways to apply the chemical nucleation rate (38) or $(70)$, which includes the unknown effective rate constants $k_{i}$, to actual problems if the condition (84) is confirmed to be satisfied. Although the confirmation requires the knowledge of the rate constants of all elementary reactions in principle, what is required is just to check the inequality like (84), which does not always need their precise values.

\section{Concluding remarks}

We have developed a kinetic theory of chemical nucleation. The steady nucleation rate (38) will provide a basis of studying astrophysical dust formation, which is usually associated with chemical reactions in the absence of monomers having the same composition as the condensates. It is of course required in the applications to actual situations to take into account of various other effects, for example, the non-LTE effect as discussed by Gail \& Sedlmayr (1988), Kozasa et al. (1996) and Patzer et al. (1998) for the circumstellar condensation, and by Yamamoto (1985) for condensation and sublimation in the protoplanetary disk. The present theory has focused on the aspect of chemical reactions in nucleation phenomena, and has derived the nucleation rate without approximation, which we hope has a wide applicability not only in astrophysics but also in other fields. For practical applications and comparisons with experiments, one needs to estimate the rate constants $k_{i}$ appearing in Eq. (38) or $k_{i_{*}}$ in Eq. (70). Since it is difficult in general to estimate $k_{i}$ or $k_{i_{*}}$ itself, the effective approximations should be sought. The key-species approximation discussed in Sect. 6 is one of those approximations valid if the condition (84) is fulfilled.

There have been two definitions of equilibrium in formulating the nucleation rate. The conventional one is a metastable supersaturated-state of a vapor with the absence of a bulk condensate (e.g., Feder et al. 1966; Abraham 1974), and the other is a saturated state of a vapor coexisting with a bulk condensate (Katz \& Wiedersich 1977). We have employed the view of Katz \& Wiedersich (1977) and Patzer et al. (1998). Although either of the definitions can be employed so far as the consistency in the theory is preserved, the latter one is clearer because of its reality and stability. The quantities in equilibrium such as the equilibrium concentrations are those for $S=1$ in the present definition of equilibrium.

It should be pointed out that the present formulation does not depend on a particular cluster model, so is free from the debate on the evaluation of the Gibbs free energy change in the cluster formation. The expression of the minimum work given by Eqs. (41) or (44), which equals the Gibbs free energy change if the clusters and the vapor are of equal temperature and pressure, is a general expression comprised of the volume energy $-(i-1) \ln S$ and the "surface energy". Note that this expression is independent of any cluster model. The "surface energy" term includes only one unknown quantity, $\chi(i)$, the minimum work needed to form an $i$-mer at a standard pressure $P_{0}$. The evaluation of $\chi(i)$ is a subject of the debate, which we have not discussed in this paper. Once $\chi(i)$ is known, the nucleation rate $J$ is straightforwardly calculated from Eq. (38), or more simply from the approximate Eq. (70) if only $\chi\left(i_{*}\right)$ and $\chi^{\prime \prime}\left(i_{*}\right)$ are known together with the critical size $i_{*}$. 
Acknowledgements. The authors thank C. Kaito for organising a workshop on the Physics of Nanostructure at Ritsumeikan University, and K. K. Tanaka for fruitful discussion. They thank K. Ikeda and Y. Saito for invaluable comments at the workshop. They also thank B. Patzer for her comments, which helped improving the manuscript. One of the authors (T.C.) acknowledges support from the Japanese Society for the Promotion of Science (JSPS). This work was supported by the grant from the Institute of Low Temperature Science, Hokkaido University, Advisory Committee (proposal Nos. 00-47, 01-50), and by the grants-in-aid (09640528, 13440059) from the JSPS.

\section{Appendix A: Proof of the fact that $\alpha \mathcal{y} / \mathrm{k}_{\mathcal{X}}$ is independent of the concentrations.}

The proof is given by induction. For $n=1$, the reaction is an elementary reaction, thus the ratio $\alpha \mathcal{y} / k_{\mathcal{X}}$ is independent of the concentrations by the postulate stated in Sect. 2.1. For an illustrative purpose, let us examine the case of $n=2$, for which the steady currents $J$ for the elementary reactions (4) and (5) are given by

$$
\begin{aligned}
J & =k_{1} X B_{1}-\alpha_{1} Z_{1} D_{11} \cdots \\
& =k_{2} Z_{1} B_{2}-\alpha_{2} Y D_{21} \cdots
\end{aligned}
$$

Eliminating the concentration $Z_{1}$, one obtains the steady current for the overall reaction as

$J=k_{\mathcal{X}} X B_{1} B_{2}-\alpha_{\mathcal{Y}} Y F_{1} \cdots$

with

$k_{\mathcal{X}}=\frac{k_{1} k_{2}}{k_{2} B_{2}+\alpha_{1} D_{11} \cdots}, \quad \alpha \mathcal{Y}=\frac{\alpha_{1} \alpha_{2}}{k_{2} B_{2}+\alpha_{1} D_{11} \cdots}$.

The ratio of the effective rate constants given by

$\frac{\alpha \mathcal{Y}}{k_{\mathcal{X}}}=\frac{\alpha_{1} \alpha_{2}}{k_{1} k_{2}}$

is expressed only by the rate constants of the elementary reactions, thus is independent of the concentrations $B_{1}, B_{2}$ and $F_{1}, \cdots$.

Now, assume that $\alpha / k$ in the steady current for $n=p$ given by

$J=k X B_{1} \cdots B_{p}-\alpha Y D_{11} \cdots$

for the overall reaction

$\mathcal{X}+\mathcal{B}_{1}+\cdots+\mathcal{B}_{p} \rightleftharpoons \mathcal{Y}+\mathcal{D}_{11}+\cdots$

is concentration-independent. Splitting the overall reaction for $n=p+1$ expressed by

$\mathcal{X}+\mathcal{B}_{1}+\cdots+\mathcal{B}_{p}+\mathcal{B}_{p+1} \rightleftharpoons \mathcal{Y}+\mathcal{F}_{1}+\cdots$

into the following sequential reactions such as

$$
\begin{aligned}
\mathcal{X}+\mathcal{B}_{1}+\cdots+\mathcal{B}_{p} & \rightleftharpoons \mathcal{Z}_{p}+\mathcal{D}_{11}+\cdots \\
\mathcal{Z}_{p}+\mathcal{B}_{p+1} & \rightleftharpoons \mathcal{Y}+\mathcal{D}_{21}+\cdots
\end{aligned}
$$

one has the steady current as

$$
\begin{aligned}
J & =k_{1} X B_{1} \cdots B_{p}-\alpha_{1} Z_{p} D_{11} \cdots \\
& =k_{2} Z_{p} B_{p+1}-\alpha_{2} Y D_{21}
\end{aligned}
$$

according to Eq. (8). Elimination of $Z_{p}$ leads to

$J=k_{\mathcal{X}} X B_{1} \cdots B_{p+1}-\alpha \mathcal{Y} Y F_{1} \cdots$

with

$$
\begin{aligned}
k_{\mathcal{X}} & =\frac{k_{1} k_{2}}{k_{2} B_{p+1}+\alpha_{1} D_{11} \cdots} \\
\alpha_{1} \alpha_{2} & =\frac{\alpha_{2}}{k_{2} B_{p+1}+\alpha_{1} D_{11} \cdots} .
\end{aligned}
$$

Thus one has

$\frac{\alpha_{\mathcal{Y}}}{k_{\mathcal{X}}}=\frac{\alpha_{1}}{k_{1}} \cdot \frac{\alpha_{2}}{k_{2}}$.

According to the assumption for $n=p, \alpha_{1} / k_{1}$ is independent of the concentrations of $B_{1}, \cdots, B_{p}$ and $F_{1}, \cdots$, and $\alpha_{2} / k_{2}$ is also concentration-independent because the reaction (A.10) is an elementary reaction. Thus, the ratio $\alpha \mathcal{Y} / k_{\mathcal{X}}$ is concentration-independent for $n=p+1$ as well.

\section{Appendix B: Proof of Eq. (21)}

Although one can prove Eq. (21) by substituting Eq. (23) into the LHS of Eq. (21), we give here a simple proof.

Consider the equilibrium between $i$-mers and a bulk condensate such as

$\mathcal{A}_{i} \rightleftharpoons i \mathcal{A}_{\mathrm{s}}$,

then the equilibrium concentration of the $i$-mers is expressed by

$\dot{c}(i)=\frac{P_{0}}{k_{\mathrm{B}} T} \exp \left[-\frac{\chi(i)-i \mu_{\mathrm{s}}}{k_{\mathrm{B}} T}\right]$

from the equilibrium condition $\dot{\mu}(i)=i \mu_{\mathrm{s}}$. Using Eq. (44), we have

$$
\begin{aligned}
S^{i} \dot{c}(i) & =\frac{P_{0}}{k_{\mathrm{B}} T} \exp \left[i \ln S-\frac{\chi(i)-N \mu_{\mathrm{s}}}{k_{\mathrm{B}} T}\right] \\
& \simeq \frac{P_{0}}{k_{\mathrm{B}} T} \exp [-g(i)] \quad(i \gg 1) .
\end{aligned}
$$

For $S>1, \lim _{i \rightarrow \infty} g(i)=-\infty$ results from Eq. (45). Thus, since $c(i)$ is necessarily finite, Eq. (21) has been proved.

\section{References}

Abraham, F. F. 1974, Homogeneous nucleation theory (Academic Press, New York)

Chigai, T., Yamamoto, T., \& Kozasa, T. 1999, ApJ, 510, 999

Feder, J., Russell, K. C., Lothe, J., \& Pound, G. M. 1966, Adv. Phys., 15, 111

Gail, H.-P., \& Sedlmayr, E. 1988, A\&A, 206, 153 
Girshick, S. L. 1997, J. Chem. Phys., 107, 1948

Hirschfelder, J. O. 1974, J. Chem. Phys., 61, 2690

Katz, J. L., \& Wiedersich, H. 1977, J. Colloid. Interface Sci., 61,351

Kitahara, K. 1994, Science of nonequilibrium systems II - Statistical physics of relaxation processes (Kodansha, Tokyo)

Kozasa, T., Dorschner, J., Henning, T., \& Stognienko, R. 1996, A\&A, 307, 551

Kozasa, T., \& Hasegawa, H. 1987, Prog. Theor. Phys., 77, 1402

Landau, L. D., \& Lifschitz, E. M. 1980, Statistical physics (3rd edition), Part 1 (Butterworth-Heinemann, Oxford)
McQuarrie, D. A., \& Simon, J. D. 1997, Physical chemistry, A Molecular approach (University Science Books, Sausalito) Nuth, J. A., Rietmeijer, F. J. M., Hallenbeck, S. L., Withey, P. A., \& Ferguson, F. 2000, in Thermal Emission Spectroscopy and Analysis of Dust, Disks, and Regoliths, ed. M. L. Sitko, A. L. Sprague \& D. K. Lynch, ASP Conf. Ser., 196, 313

Patzer, A. B. C., Gauger, A., \& Sedlmayr, E. 1998, A\&A, 337, 848

Rice, H. 1950, J. Chem. Phys., 18, 840

Sedlmayr, E., \& Dominik, C. 1995, Space Sci. Rev., 73, 211

Yamamoto, T. 1985, A\&A, 142, 31 